\title{
Route to calculate the length scale for the glass transition in polymers
}

\author{
D. Cangialosi, ${ }^{1, *}$ A. Alegría, ${ }^{2,3}$ and J. Colmenero ${ }^{1,2,3}$ \\ ${ }^{1}$ Donostia International Physics Center, Paseo Manuel de Lardizabal 4, 20018 San Sebastián, Spain \\ ${ }^{2}$ Departemento de Física de Materiales, Universidad del País Vasco (UPV/EHU), Apdo. 1072, 20080 San Sebastián, Spain \\ ${ }^{3}$ Unidad de Física de Materiales, Centro Mixto CSIC-UPV, Apdo. 1072, 20080 San Sebastián, Spain
}

(Received 28 November 2006; published 27 July 2007)

\begin{abstract}
The occurrence of glass transition is believed to be associated to cooperative motion with a growing length scale with decreasing temperature. We provide a route to calculate the size of cooperatively rearranging regions (CRR) of glass-forming polymers combining the Adam-Gibbs theory of the glass transition with the selfconcentration concept. To do so we explore the dynamics of glass-forming polymers in different environments. The material specific parameter $\alpha$ connecting the size of the CRR to the configurational entropy is obtained in this way. Thereby, the size of CRR can be precisely quantified in absolute values. This size results to be in the range $1-3 \mathrm{~nm}$ at the glass transition temperature depending on the glass-forming polymer.
\end{abstract}

DOI: $10.1103 /$ PhysRevE.76.011514

PACS number(s): 64.70.Pf, 77.22.Gm, 83.80.Rs, 83.80.Tc

\section{INTRODUCTION}

The nature of the glass transition is one of the most important unsolved problems in condensed matter physics and research in this field has enormously intensified in the last decades due to its strong fundamental as well as applicative implications. Among the peculiar phenomena displayed by glass-forming liquids, the super-Arrhenius temperature dependence of the viscosity and the structural correlation time is certainly one of the most intriguing. In this framework, more than 40 years ago Adam and Gibbs [1] theorized that such a pronounced temperature dependence of the structural correlation time is due to a cooperative process involving several basic structural units forming cooperatively rearranging regions (CRR), which size increases with decreasing temperature. Since then a great deal of theoretical approaches $[2,3]$ as well as simulation studies [4] and very recently experimental studies employing multipoint dynamical susceptibilities [5] have been devoted in the search of good candidates for CRR as well as their size and temperature dependence. All of these studies suggest that a growing correlation length with decreasing temperature of the order of several nanometers exists. According to the Adam-Gibbs (AG) theory of the glass transition, the increase of the structural relaxation time with decreasing temperature, accompanied by the growth of the cooperative length scale, is due to the decrease of the number of configurations the glass former can access, namely the configurational entropy $\left(S_{c}\right)$ of the system. The connection between the structural relaxation time and $S_{c}$ is expressed by [1]

$$
\tau=\tau_{0} \exp \left[C /\left(T S_{c}\right)\right],
$$

where $C$ is a glass former specific temperature independent parameter and $\tau_{0}$ is the pre-exponential factor. The dynamics of a large number of glass-forming systems have been successfully described through the AG equation in both experiments for low molecular weight glass formers [6] and polymers [7], as well as simulations [8]. Recent studies on

*Corresponding author; Email address: swxcacad@sw.ehu.es polymerizing systems also provide a successful application of the AG equation [9]. Apart from the relation between the relaxation time and the configurational entropy, the AG theory provides a connection between the number of basic structural units belonging to the CRR and the configurational entropy, $N \approx S_{c}^{-1}$. Several recent simulation studies, where the cooperative length scale and the configurational entropy were obtained within the framework of the stringlike motion and the potential energy landscape of the glass former, respectively, successfully tested the validity of this correlation [10]. Being the number of particles proportional to the volume of the CRR, the characteristic length scale $\xi$ can be related to the configurational entropy by

$$
\xi / 2=r_{c}=\alpha S_{c}^{-1 / 3},
$$

where $r_{c}$ is the equivalent radius of the CRR and $\alpha$ is a material specific parameter. Here, the exponent $-1 / 3$ implies that the fractal dimension for the growth of CRR equals 3. This value means that CRR possess a compact shape growing in three directions. This hypothesis has been rationalized within the framework of the random first-order transition theory [11]. Very recent molecular dynamics simulations in a Lennard-Jones liquid, performed taking into account the motion of all particles [12], also suggest that the structural rearrangement involves the motion of compact regions. Despite the ability to describe the temperature dependence of the structural relaxation and predict an increasing length scale with decreasing temperature, the glass-former specific parameter $\alpha$ is not reliably obtained from the AG theory as highlighted by several authors $[3,13]$. Hence, the size of the CRR cannot be estimated a priori from the AG theory alone. In this work we apply the AG theory to polymer blends and polymer-mixture systems in order to provide a new route to precisely determine the parameter $\alpha$ and hence the size of CRR. To do this, we incorporate in the AG theory the concept of self-concentration first proposed by Chung and Kornfield [14] and later developed by Lodge and McLeish [15] to describe the segmental dynamics of miscible polymer blends. This approach has been recently exploited by us to describe the dynamics of miscible polymer blends and con- 


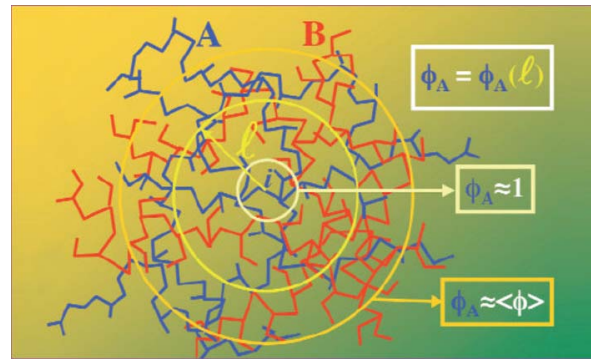

FIG. 1. (Color online) Schematic illustration of the selfconcentration concept.

centrated polymer solutions and provided an accurate description of the component segmental dynamics (the $\alpha$ relaxation) of these systems [16]. The essential features of the concept of self-concentration are explained in Fig. 1 and can be summarized as follows: when a volume is centered on the basic structural unit of one of the polymers of a given mixture, the effective concentration $\left(\phi_{\text {eff }}\right)$ will be in general different from the macroscopic one. Two extreme cases are possible: (i) if this volume equals the volume of the basic structural unit, then $\phi_{\text {eff }}=1$; (ii) if, on the other hand, this volume is large enough, $\phi_{\text {eff }}$ will be equal to the macroscopic concentration, $\phi_{\text {eff }}=\phi$. The effective concentration is related to the self-concentration $\left(\phi_{s}\right)$ and to the macroscopic concentration through [15], $\phi_{\text {eff }}=\phi_{s}+\left(1-\phi_{s}\right) \phi$. The selfconcentration, namely the volume fraction occupied by the isolated chain containing the unit of reference, can be related to the radius of the volume through simple geometric considerations involving the Kuhn and the packing lengths [17]. It is worth remarking that, as the volume of CRR is expected to be neither large enough to make the concentration equal to the macroscopic one nor equal to that of the monomer ( $\phi$ $<\phi_{\text {eff }}<1$ ) [15-17], the component dynamics will be intermediate between that of the pure polymer and the average structural dynamics of the mixture. This means that the selfconcentration concept constitutes an extremely sensitive tool when exploring length scales of the order of those expected for CRR. Furthermore, the prominent role of selfconcentration is in agreement with the hypothesis of a characteristic length scale for the glass transition.

In order to apply the AG theory to mixtures, $S_{c}, C$, and $\tau_{0}$ in Eqs. (1) and (2) must be evaluated in the cooperative volume. This means that these quantities are functions of the effective concentration of the polymer under examination. In our previous works, we found that a linear combination of the parameters of the pure components of the mixture through the effective concentration allows a good description of dynamics data [16],

$$
X=\phi_{\text {eff } A} X_{A}+\left(1-\phi_{\text {eff } A}\right) X_{B},
$$

where $X$ is either $S_{c}, C$ or $\ln \tau_{0}$. Due to the experimental inaccessibility of the configurational entropy, we have exploited the proportionality of this quantity with the excess entropy, namely the entropy of the liquid in excess to that of the corresponding crystal. However, it is worth noticing that the proportionality constant between the two magnitudes is unlikely to be the same for both components of the mixtures. This means that this proportionality constant will be dependent on the effective concentration in most of the cases and the straightforward employment of the excess entropy instead of the configurational entropy is not rigorously correct. However, it was found that the effective concentration only varies with temperature by less than 0.1 for all investigated mixtures at fixed macroscopic concentration. In this range of variation, the change of the proportionality constant between excess and configurational entropy can always be substantially neglected and, therefore, the excess entropy can still be used to fit data. An alternative approach would be that followed by us in some recent papers [18], where the configurational entropy was directly obtained for all components of the mixtures employing the approach of Di Marzio and Dowell [19] or using available literature data [20].

Equations (1), (2), and (3) can be fitted to experimental data having $\alpha$ as the only unknown parameter. As this is a glass-former specific parameter, the presence of the other component in CRR can in principle affect the value of $\alpha$. This has been found to be the case especially for polymersolvent systems $[16,18]$. We have overcome this problem by studying the dynamics of polymer-polymer and polymersolvent mixtures at elevated concentration of the polymer for which $\alpha$ is being calculated. This allows a straightforward extrapolation of the $\alpha$ parameter to the pure polymer. Furthermore, it is noteworthy that for concentrated polymer mixtures both concentrations fluctuations, which might affect the dynamics, and deviations from ideality, which would induce the failure of Eq. (3), are minimized. We have thus obtained values of $\alpha$ for polystyrene (PS), poly(vinyl acetate) (PVAc) and used those already obtained for poly(ochlorostyrene) (PoClS) [16], poly(vinyl methyl ether) (PVME) [16], polyisoprene (PI) [18], and poly(vinylethylene) (PVE) [18]. In such a way we have investigated six typical glass-forming polymers with different rigidity. We have found that a single polymer specific value of $\alpha$ is obtained when extrapolating to $100 \%$ concentration independently on the environment surrounding the polymer under consideration. The achievement of a temperature independent $\alpha$ parameter results, via Eq. (3), in an increasing length scale for structural relaxation with decreasing temperature of the order of nanometers. In particular, the resulting size of CRR was between 1 and $3 \mathrm{~nm}$ depending on the characteristics of the polymer. For a given polymer in the accessible temperature range the diameter of CRR only varies by about $20 \%$.

\section{EXPERIMENT}

PVAc, poly(2,6-dimethyl-1,4-phenylene oxide) (PPhO), ortho-terphenyl (OTP), and toluene were purchased by Sigma-Aldrich. The molecular weight of the two polymers were, respectively, $M_{w}=83000 \mathrm{~g} / \mathrm{mol} \quad\left(M_{w} / M_{m}=4.2\right)$ and $M_{n}=23000 \mathrm{~g} / \mathrm{mol}\left(M_{w} / M_{n}=2.3\right)$. PS was purchased from Polymer Source Inc. The molecular weight was $M_{n}=70400 \mathrm{~g} / \mathrm{mol} \quad\left(M_{w} / M_{n}=1.04\right)$ Bis-phenol-Cdimethylether (BCDE) was synthesized as describe elsewhere [21]. Highly concentrated PS and PVAc in different 
TABLE I. Relevant parameters for investigated polymers. Data for the $C$ parameter of the AG relation, the parameters $a$ and $b$ of the excess specific heat and $\alpha$ are referred to on a per mole of monomer basis.

\begin{tabular}{lcccccc}
\hline \hline & PVME & PVAc & PS & PoClS & PI & PVE \\
\hline$T_{g}(\mathrm{~K})$ & 249 & 304 & 373 & 402 & 213 & 263 \\
$T_{K}(\mathrm{~K})$ & 203.5 & 256 & 322 & 340 & 167 & 228 \\
$\log _{10} \tau_{0}$ & -13.0 & -13.8 & -12.4 & -12.9 & -13 & -10.6 \\
$C\left(\mathrm{~J} \mathrm{~mol}^{-1} \mathrm{~K}^{-1}\right)$ & 21900 & 41450 & 26400 & 61800 & 20400 & 6400 \\
$a\left(\mathrm{~J} \mathrm{~mol}^{-1} \mathrm{~K}^{-1}\right)$ & 68.3 & 105.3 & 99.0 & 98.4 & 60.2 & 28.3 \\
$b\left(\mathrm{~J} \mathrm{~mol} \mathrm{~K}^{-1} \mathrm{~K}^{-1}\right)$ & -0.16 & -0.21 & -0.17 & -0.17 & -0.14 & -0.02 \\
$l_{k}(\AA)$ & 13 & 17 & 18 & 17.3 & 8.2 & 14 \\
$l_{p}(\AA)$ & 2.7 & 3.7 & 3.9 & 3.9 & 3.2 & 2.8 \\
$\alpha\left(\mathrm{J}^{1 / 3} \mathrm{~mol}^{-1 / 3} \mathrm{~K}^{-1 / 3}\right)$ & $10.7 \pm 0.5$ & $17 \pm 0.5$ & $19.5 \pm 0.5$ & $24 \pm 2$ & $17.5 \pm 0.5$ & $11 \pm 0.5$ \\
\hline \hline
\end{tabular}

environments were investigated by means of broadband dielectric spectroscopy (BDS). PVAc and PS dynamics were investigated, respectively, in toluene and BCDE, and OTP and $\mathrm{PPhO}$. Dielectric measurements were carried out using a high precision dielectric analyzer (ALPHA, Novocontrol $\mathrm{GmbH}$ ) and a Novocontrol Quatro cryosystem for temperature control with a precision of $0.1 \mathrm{~K}$. Measurements were performed over a wide frequency $\left(10^{-2}-10^{6} \mathrm{~Hz}\right)$ and temperature range in isothermal steps starting for the highest temperature. As a general rule the relevant relaxation time at any temperature was obtained from dielectric relaxation spectra as the reciprocal of the angular frequency at the maximum of the permittivity loss. Structural relaxation data for toluene [22], BCDE [23], OTP [24], and PPhO [25] were taken from the literature. Calorimetric measurements were carried out on pure PVAc, PS, and BCDE by means of the differential scanning calorimeter (DSC-Q1000) from TAInstruments. Measurements were performed in a temperature modulated mode with average heating rate of $0.1 \mathrm{~K} / \mathrm{min}$ and amplitude of $0.3 \mathrm{~K}$. Different oscillation frequencies were investigated and the so-obtained specific heats were extrapolated to zero frequency in order to obtain quasistatic values of the specific heat itself. Specific heats of toluene and OTP [26], and PPhO [27] were taken from the literature. Since the configurational entropy cannot be determined experimentally, the entropy of the liquid in excess to the corresponding crystal was used exploiting the proportionality between the two magnitudes [7]. This only scales the value of $C$ in Eq. (1) and $\alpha$ in Eq. (3). A linear form of the excess specific heat, $\Delta C_{p}=C_{p}^{\text {melt }}-C_{p}^{\text {crystal }}$, where $a$ and $b$ are material specific constants, was employed to obtain the excess entropy [28]. This was obtained integrating the relation $\int_{T_{K}}^{T}\left[\Delta C_{p}(T) / T\right] d T$; where $T_{K}$ is the Kauzmann temperature, namely the temperature where the entropy of the liquid equals that of the crystal. Due to the lack of crystalline specific heat data for glassforming polymers, the latter quantity was determined identifying it with the temperature where the dielectric relaxation time of the $\alpha$ process tends to diverge [6]. Hence, the knowledge of the parameters $a$ and $b$ allows fitting pure components dynamics data through the $\mathrm{AG}$ equation (dashed line in Fig. 3 for pure PVAc) to obtain $C, \ln \tau_{0}$, and $T_{K}$. The values of the parameters $a$ and $b$ together with $T_{g}, T_{K}, C$, and $\log _{10} \tau_{0}$ are summarized in Table I for the pure polymers.
Literature values of the packing $\left(l_{p}\right)$ and Kuhn $\left(l_{k}\right)$ length, relating the size of CRR to the self-concentration [17], are also included. In addition, previously obtained BDS and calorimetric data for PVAc in poly(ethylene oxide) [29], for PoClS in PVME and low molecular weight PS [16] and PVME in toluene, PoClS and PS with various molecular weight [16] were employed.

\section{RESULTS AND DISCUSSION}

In this section, we present the results obtained for mixtures at high concentration of PVAc as a showcase and illustrate the methodology followed to evaluate the parameter $\alpha$ for this polymer. Figure 2 shows the dielectric loss versus frequency for PVAc at various concentrations of toluene and fixed temperature. The increase in toluene concentration clearly provokes a speed-up of PVAc dynamics. Similar results were obtained for PVAc in PEO and BCDE. The average relaxation time of PVAc in mixtures with toluene as well as that of pure PVAc as a function of the inverse temperature is displayed in Fig. 3. The continuous lines represent the best fits of the proposed model to experimental data. The quality of the fits is excellent. Furthermore, the hypothesis of a dimensionality for the growth of CRR lower than 3, as e.g., in

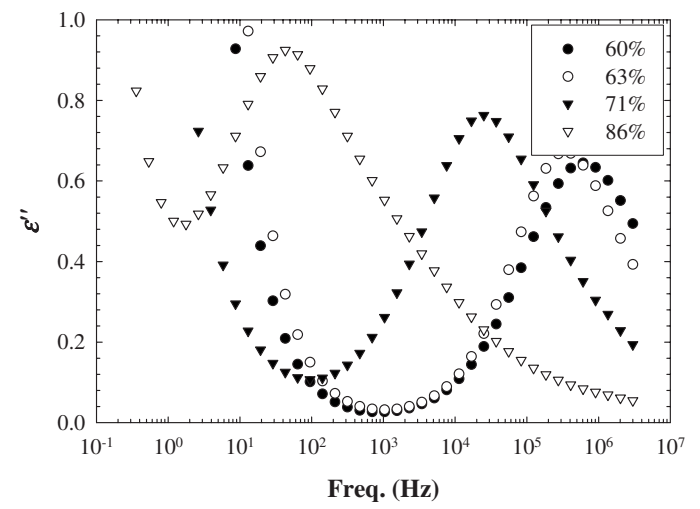

FIG. 2. Permittivity loss vs logarithm of the frequency for PVAc/toluene system at $308 \mathrm{~K}$ and the following weight percentages of PVAc, $60 \%$ (filled circles), $63 \%$ (empty circles), $71 \%$ (filled triangles), and $86 \%$ (empty triangles). 


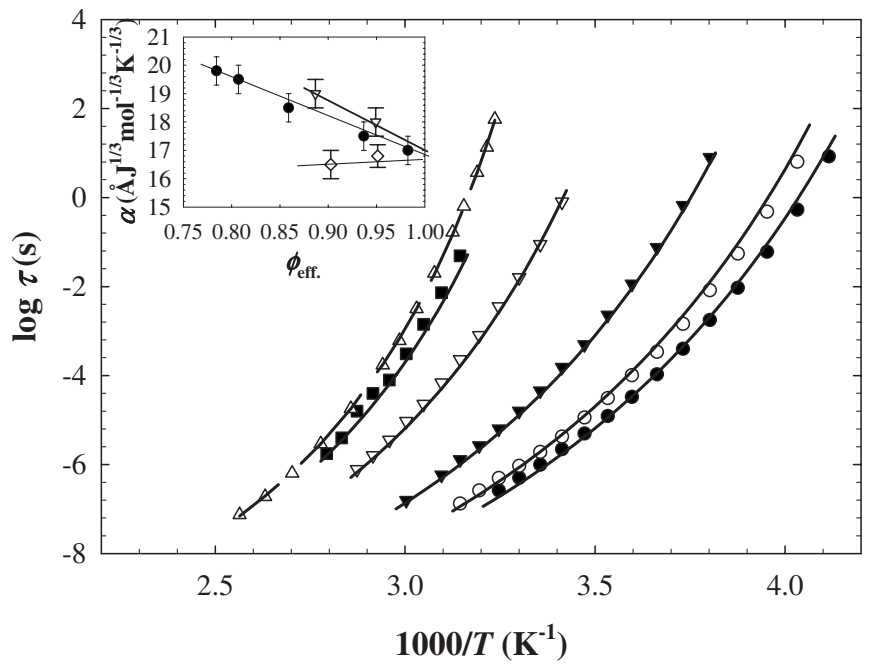

FIG. 3. Logarithm of the relaxation time vs temperature for PVAc segmental dynamics in PVAc/toluene systems with the following PVAc weight percentages, 60\% (filled circles), 63\% (empty circles), $71 \%$ (filled down triangles), $86 \%$ (empty down triangles), and $95 \%$ (filled square). Pure PVAc segmental dynamics data are also shown (empty up triangles). The solid lines are the fits of the model to PVAc segmental dynamics in toluene solutions and the dashed line is the fit through the AG equation to pure PVAc dynamics data. The inset of the figure represents the variation of the $\alpha$ parameter with the average effective concentration of PVAc in toluene (filled circles), BCDE (empty triangles), and PEO (empty circles). The solid lines are a linear fit of experimental data, which, extrapolating to pure PVAc, allow the evaluation of the $\alpha$ parameter for pure PVAc.

stringlike motion [4], would result in a poorer quality of the fits. This would suggest that CRR actually grow in three dimensions at temperature close to $T_{g}$. Analogously, the fits performed for the dynamics of PVAc in mixture with PEO and BCDE resulted in a successful description of the data. The parameter $\alpha$ obtained from the best fits of our data is plotted in the inset of Fig. 3 as a function of the average effective concentration. Strikingly the extrapolation to pure PVAc leads to a single value of $\alpha$ independently of the environment surrounding PVAc chains. This can be regarded as the intrinsic $\alpha$ connecting PVAc configurational entropy with its size of CRR. The same procedure was applied to evaluate the parameter $\alpha$ for PS investigating its dynamics in OTP and PPhO and similarly to PVAc, a single $\alpha$ was obtained. Furthermore, a single value of $\alpha$ was obtained for PoClS [16], PVME [16], PI [18], and PVE [18] studying their dynamics in various environments. The so-obtained values of $\alpha$ are tabulated in Table I.

The availability of the polymer specific parameter $\alpha$ allows determining via Eq. (3) the size of CRR and its temperature variation, starting from the knowledge of the excess entropy determined from calorimetric measurements. The soobtained diameter of CRR is displayed in Fig. 4 as a function of temperature for the investigated polymers. Though the size of CRR clearly depends on temperature, its variation is rather smooth $(\approx 20 \%$ for the diameter $)$. However, we note that this can translate in a dramatic change of the number of particles involved in the cooperative rearrangement. Further-

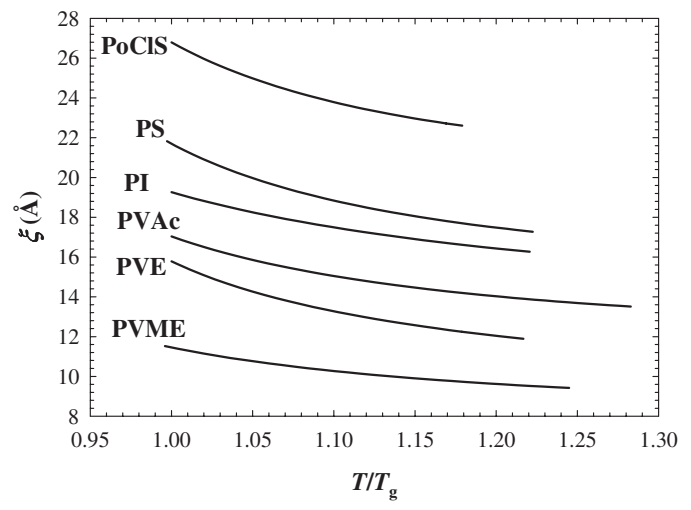

FIG. 4. Size of CRR vs the temperature normalized at the $T_{g}$ for all investigated polymers.

more, a clear relation with the rigidity of the chemical structure can be observed, being the size of CRR larger for the most rigid polymer, namely PoClS, and smaller for the most flexible, namely PVME. The only exception to this correlation seems to be PI, which presents relatively large sizes of CRR despite its flexibility. Remarkably the addition of one bulky chlorine atom in the phenyl ring of PS results in a substantial increase in the size of CRR for the resulting polymer, namely PoClS. These results provide a positive indication of the reliability of the approach followed. The dependence of the size of CRR on the rigidity of the polymer suggests a positive correlation between the size itself and the Kuhn segment, shown in Table I. The assessment of the size of CRR allows performing a rough estimation of the number of particles involved in the cooperative rearrangement. This can be done considering the interchain distance for the investigated glass-forming polymers, which can be obtained from the static structure factor $S(Q)$. Values for this distance can be calculated from the available measurements of the static structure factor of PS [30,31], PVAc [29], PVME [32], PI [33], and PVE [34], being the resulting values, $d_{\mathrm{PS}}=9 \AA$, $d_{\mathrm{PVAc}}=8.4 \AA, d_{\mathrm{PVME}}=6.3 \AA, d_{\mathrm{PI}}=4.8 \AA$, and $d_{\mathrm{PVE}}=6.6 \AA$. It is noteworthy that, except for PI, the diameters of CRR found at $T_{g}$ for these polymers are almost quantitatively 2 times the corresponding intermolecular distance, suggesting that the cooperative rearrangement only involves the first shell around a basic structural unit. This would imply that the number of particles involved in each CRR at $T_{g}$ is of the order of tens. The possible connection between the size of CRR and the intermolecular distance opens interesting scenarios in the rationalization of the length scale involved in the structural relaxation of glass formers, which needs to be further explored.

Finally, it is worth comparing the sizes of CRR obtained here with the AG approach with those previously estimated employing different approaches. The size of CRR obtained for PVAc at $T_{g}, \xi \approx 1.7 \mathrm{~nm}$ agrees almost quantitatively with the characteristic length scale obtained by Berthier et al. [5] by means of multipoint dynamical susceptibility $(\xi \approx 2 \mathrm{~nm})$. The size of CRR obtained by us for PS is also in qualitative agreement with that estimated by Hempel et al. [35] in the framework of Donth approach [2] $(\xi \approx 2.2 \mathrm{~nm}$ vs $\xi \approx 3 \mathrm{~nm})$ and in excellent agreement with that estimated by Sills et al. 
[36] by means of nanoscale sliding friction $(\xi \approx 2.1 \mathrm{~nm})$. Our results are also compatible with the absence of finite size effects in confinement down to $5 \mathrm{~nm}$ [37].

\section{CONCLUSIONS}

To summarize, we have taken advantage of the concept of self-concentration in combination with the AG theory of the glass transition to open a new route to obtain the size of CRR and its temperature dependence for several glass-forming polymers. The approach proposed allows the unambiguous derivation of the polymer specific parameter $\alpha$ connecting the size of CRR to the configurational entropy. Once $\alpha$ is obtained for each polymer, the size of CRR in a wide temperature range can be determined from the knowledge of the configurational entropy of the pure polymer. The temperature variation of the diameter of CRR for a given glass-forming polymer results to be of only about $20 \%$ in the accessible temperature range. Remarkably, the values of the diameter of CRR at $T_{g}$ for the investigated polymers closely correlate with both the Kuhn length and the interchain distance.

\section{ACKNOWLEDGMENTS}

The authors acknowledge the University of the Basque Country and Basque Country Government (Contract No. 9/UPV 00206.215-13568/2001) and Spanish Minister of Education (Contract No. MAT 2004-01617) for their support. The support of the European Community within the SoftComp program is also acknowledged.
[1] G. Adam and J. H. Gibbs, J. Chem. Phys. 43, 139 (1965).

[2] E. Donth, J. Non-Cryst. Solids 53, 325 (1982).

[3] X. Y. Xia and P. G. Wolynes, Proc. Natl. Acad. Sci. U.S.A. 97, 2990 (2000).

[4] C. Donati, J. F. Douglas, W. Kob, S. J. Plimpton, P. H. Poole, and S. C. Glotzer, Phys. Rev. Lett. 80, 2338 (1998).

[5] L. Berthier, G. Biroli, J. P. Bouchaud, L. Cipelletti, D. El Masri, D. L'Hote, F. Ladieu, and M. Pierno, Science 310, 1797 (2005).

[6] R. Richert and C. A. Angell, J. Chem. Phys. 108, 9016 (1998); D. Prevosto, S. Capaccioli, M. Lucchesi, and P. Rolla, J. NonCryst. Solids 351, 2611 (2005).

[7] D. Cangialosi, A. Alegría, and J. Colmenero, Europhys. Lett. 70, 614 (2005); G. A. Schwartz, J. Colmenero, and A. Alegría, Macromolecules 39, 3931 (2006).

[8] S. Sastry, Nature (London) 409, 164 (2001); S. Mossa, E. La Nave, H. E. Stanley, C. Donati, F. Sciortino, and P. Tartaglia, Phys. Rev. E 65, 041205 (2002).

[9] S. Corezzi, D. Fioretto, and P. Rolla, Nature (London) 420, 653 (2002); S. Corezzi, D. Fioretto, and J. M. Kenny, Phys. Rev. Lett. 94, 065702 (2005).

[10] N. Giovambattista, S. V. Buldyrev, F. W. Starr, and H. E. Stanley, Phys. Rev. Lett. 90, 085506 (2003); Y. Gebremichael, M. Vogel, M. N. J. Bergroth, F. W. Starr, and S. C. Glotzer, J. Phys. Chem. B 109, 15068 (2005).

[11] J. D. Stevenson, J. Schmalian, and P. G. Wolynes, Nat. Phys. 2, 268 (2006).

[12] G. A. Appignanesi, J. A. Rodriguez Fris, R. A. Montani, and W. Kob, Phys. Rev. Lett. 96, 057801 (2006).

[13] H. Huth, M. Beiner, and E. Donth, Phys. Rev. B 61, 15092 (2000).

[14] G. C. Chung, J. A. Kornfield, and S. D. Smith, Macromolecules 27, 964 (1994).

[15] T. P. Lodge and T. C. M. McLeish, Macromolecules 33, 5278 (2000).

[16] D. Cangialosi, G. A. Schwartz, A. Alegría, and J. Colmenero, J. Chem. Phys. 123, 144908 (2005); D. Cangialosi, A. Alegría, and J. Colmenero, Macromolecules 39, 448 (2006); G. A. Schwartz, D. Cangialosi, A. Alegría, and J. Colmenero, J. Chem. Phys. 124, 154904 (2006).

[17] R. Kant, S. K. Kumar, and R. H. Colby, Macromolecules 36, 10087 (2003).
[18] D. Cangialosi, A. Alegría, and J. Colmenero, Macromolecules 39, 7149 (2006); D. Cangialosi, A. Alegría, and J. Colmenero, J. Chem. Phys. 126, 204904 (2007).

[19] E. A. Di Marzio and F. Dowell, J. Appl. Phys. 50, 6061 (1979).

[20] G. P. Johari, J. Chem. Phys. 112, 7518 (2000).

[21] G. Meier, D. Boese, and E. W. Fischer, J. Chem. Phys. 94, 3050 (1991).

[22] A. Doss, G. Hinze, B. Schiener, J. Hemberger, and R. Bohmer, J. Chem. Phys. 107, 1740 (1997).

[23] F. Alvarez, A. Hoffmann, A. Alegría, and J. Colmenero, J. Chem. Phys. 105, 432 (1996).

[24] S. S. Chang and A. B. Bestul, J. Chem. Phys. 56, 503 (1972).

[25] C. G. Robertson and G. L. Wilkes, J. Polym. Sci., Part B: Polym. Phys. 39, 2118 (2001).

[26] O. Yamamuro, I. Tsukushi, A. Lindqvist, S. Takahara, M. Ishikawa, and M. Matsuo, J. Phys. Chem. B 102, 1605 (1998).

[27] J. Pak, M. Pyda, and B. Wünderlich, Macromolecules, 36495 (2003).

[28] The specific heat of the crystal has been replaced by that of the glass. Due to the similarity of the two quantities, calculating the excess specific heat with the specific heat of the glass instead of that of the crystal results in a negligible error. See, e.g., B. Wüünderlich, ATHAS database, http://web.utk.edu/ athas/

[29] M. Tyagi et al. (unpublished).

[30] H. Furuya, M. Mondello, H. J. Yang, R. J. Roe, R. W. Erwin, C. C. Han, and S. D. Smith, Macromolecules 27, 5674 (1994).

[31] I. Iradi, F. Alvarez, J. Colmenero, and A. Arbe, Physica B 350, 881 (2004).

[32] C. Saelee, T. M. Nicholson, and G. R. Davies, Macromolecules 33, 2258 (2000).

[33] F. Alvarez, J. Colmenero, R. Zorn, L. Willner, and D. Richter, Macromolecules 36, 238 (2003).

[34] J. Colmenero (unpublished).

[35] E. Hempel, G. Hempel, A. Hensel, C. Schick, and E. Donth, J. Phys. Chem. B 104, 2460 (2000).

[36] S. Sills, T. Gray, and R. M. Overney, J. Chem. Phys. 123, 134902 (2005).

[37] A. Serghei, M. Tress, and F. Kremer, Macromolecules 39, 9385 (2006); V. Lupascu, H. Huth, C. Schick, and M. Wubbenhorst, Thermochim. Acta 432, 222 (2005). 